\title{
An immunohistochemical study of fibronectin in human osteoarthritic and disease free articular cartilage
}

\author{
K L JONES, M BROWN, S Y ALI, AND R A BROWN
}

From the Departments of Experimental Pathology and Morbid Anatomy, Institute of Orthopaedics, University of London, Stanmore, Middlesex

SUMMARY Fibronectin is a minor component of cartilage connective tissue matrix, which is reported to accumulate in increased amounts in osteoarthritis. The presence of raised levels of fibronectin in human osteoarthritic cartilage by immunoperoxidase localisation is confirmed. Residual femoral head articular cartilage from 17 patients with osteoarthritis contained variable but substantial amounts of fibronectin. This was localised mainly in a band within the matrix of the surface zone. No significant deposits of fibronectin were found in this or any other area of the normal specimens. Intracellular fibronectin was identified in some cells of the surface zone, indicating that it was, in part, synthesised locally. The presence and distribution of locally produced fibronectin in osteoarthritic cartilage suggest that its synthesis is a response by chondrocytes to changes in the cartilage matrix.

Key words: chondrocytes, connective tissue, femoral head, immunoperoxidase, matrix.

Fibronectin is an important glycoprotein component of many extracellular matrices. In addition, it is implicated in cell adhesion, migration, morphology, and differentiation.'

Fibronectin binds to collagen, proteoglycans, and hyaluronic acid ${ }^{1-3}$ and has been identified in most tissues, including cartilage, ${ }^{+5}$ though its function in hyaline cartilage is not known. Despite early reports that it was not present in mature tissue but could initiate a change in chondrocyte phenotype ${ }^{6}$ it has now been located, in varying amounts, in a range of normal and pathological articular cartilages. ${ }^{7-1.3}$ Increased fibronectin levels were seen in osteoarthritic cartilage from human ${ }^{7}$ and canine joints. " This appears to be, in part, a result of increased production by chondrocytes and retention of newly synthesised fibronectin by the altered cartilage matrix. " Another potential source of fibronectin in articular cartilage is the synovial fluid. In rheumatoid arthritis, for example, increased concentrations of fibronectin have been reported in synovial fluid ${ }^{1+}$ with deposition of fibronectin in the synovial tissue $^{15 \mathrm{in}}$ and on the surface of the articular cartilage. Osteoarthritic synovial fluid is also rich in

Accepted for publication 17 April 1987.

Correspondence to Dr R A Brown. Experimental Pathology Unit. Institute of Orthopacdics. Stanmore. Middlesex HA7 4LP. fibronectin, with $55 \%$ higher levels than in normal human plasma. ${ }^{15}$

Immunohistochemical techniques at the light microscopic level have been used to detect fibronectin within articular cartilage of human, dog, and rabbit. ${ }^{8} 91.3$ These studies indicated that fibronectin is distributed throughout the matrix in both osteoarthritic and disease free canine and lupine cartilage. Studies on normal human cartilage have relied on single specimens from unspecified sites, and findings have been contradictory. Widespread staining of the deep zone matrix was reported in one case. whereas in the other only pericellular staining was seen. ${ }^{9}$

To clarify the role of fibronectin in human cartilage pathology we have investigated its origin and distribution within carefully selected areas of human femoral head cartilage from patients with primary and secondary osteoarthritis and from disease free cartilage.

\section{Materials and methods}

Clinical assessments of patients examined were based on medical records and radiographs. Specimens of residual (type IV) and osteophytic (type VI) cartilage as defined by $\mathrm{Ali}$ and Bayliss ${ }^{17}$ were obtained from osteoarthritic human femoral heads 
immediately after total hip replacement. Both full depth and thin sloping cartilage (type IV) were taken from the region at the margin of exposed bone. Disease free cartilage (type I) was obtained from femoral heads after amputation. In all cases full depth cartilage specimens into subchondral bone were taken from the lateral non-weight-bearing area. Specimens were fixed in $10 \%$ formalin saline and taken to wax in the customary manner for the preparation of $5 \mu \mathrm{m}$ sections.

IMMUNOHISTOCHEMISTRY: DIRECT

PEROXIDASE TECHNIQUE

Decalcified sections were placed onto Histostik (Histolab, Herts) coated slides. Dewaxed sections were digested with 500 units $/ \mathrm{ml}$ of leech hyaluronidase (Biopharm UK Ltd, Swansea) in $50 \mathrm{mM}$ sodium acetate, $100 \mathrm{mM}$ sodium chloride buffer $\mathrm{pH}$ 5 , or 0.5 units of chondroitinase ABC (Sigma, Dorset) in $250 \mu \mathrm{l}$ TRIS (tris(hydroxymethyl)methylamine) enriched buffer $\mathrm{pH} 8$ (50 mM TRIS; $650 \mathrm{mM} \mathrm{HC1} ; 65 \mathrm{mM} \mathrm{NaC1} ; 60 \mathrm{mM}$ sodium acetate; $1 \mathrm{mg} / \mathrm{ml}$ bovine serum albumin), at $37^{\circ} \mathrm{C}$ in a moist chamber. To ensure complete removal of glycosaminoglycan the most suitable enzyme conditions were determined by alcian blue staining of sections digested for increasing time periods. After washing for 10 minutes in TRIS buffered saline $(50 \mathrm{mM}$ TRIS-HC1; $0.15 \mathrm{M} \mathrm{NaC1} \mathrm{pH} 7.5$ ) sections were treated with $0.5 \%$ hydrogen peroxide in methanol for 30 minutes at room temperature to block endogenous peroxidase and washed as before. This was followed by treatment with fibronectin depleted non-immune sheep serum (Serotec, Bicester), diluted 1:5 for 20 minutes to block non-specific binding. This serum was prepared by roller mixing whole serum with gelatin-Sepharose 4B (Pharmacia) for one hour in TRIS buffered saline. Sections were then treated with sheep antihuman fibronectin immunoperoxidase conjugate (Serotec) diluted 1:50, for 30 minutes, washed with TRIS buffered saline, and stained for 40 minutes in $0.4 \mathrm{mg} / \mathrm{ml}$ aminoethylcarbazole in $0.5 \mathrm{M}$ acetate buffer, $\mathrm{pH} 5$, containing $0.03 \%$ hydrogen peroxide and $5 \%$ dimethylformamide. Washed sections were counterstained with haematoxylin and mounted in glycerol.

\section{Controls}

I. Sections were incubated with sheep antihuman fibronectin for 30 minutes before the addition of conjugate. II. Sheep antihuman fibronectin immunoperoxidase conjugate was preabsorbed by incubation with purified fibronectin. III. Endogenous peroxidase activity was checked using the staining procedure alone.
INDIRECT: FITC TECHNIQUE

Sections were prepared as above and digested wit 0.5 units of chondroitinase $A B C$ and 78 units of testicular hyaluronidase (Sigma, Dorset) in $300 \mu \mathrm{l}$ of TRIS enriched buffer $\mathrm{pH} 7$ for 40 minutes at $37^{\circ} \mathrm{C}$ After washing, sheep antihuman fibronectin wa added for 30 minutes and washed for 10 minutes This was followed by treatment with fluoresceif isothiocyanate (FITC) linked antisheep Ig(0) (Serotec, Bicester) diluted 1:50, for 30 minutes After washing for one hour sections were mounted in buffered glycerol plus 1,4-diazobicylo(2.2.240 octane $25 \mathrm{~g} / \mathrm{l}$ (Sigma, Dorset), to retard the fading of fluorescence during microscopy. ${ }^{18}$

\section{Controls}

I. Sections were incubated with 1:20 sheep norbo immune serum in place of sheep antihuman fibo ronectin. II. The addition of sheep antihuma fibronectin was omitted. III. To test for autofluorescence, FITC linked antisheep IgG was omiz ted.

\section{EXAMINATION OF SECTIONS}

Sections were examined using a Zeiss photomigroscope III fitted with an epifluorescence attachmon ne Films used were Kodak Ektachrome 50 professional tungsten film, and for the fluorescence study, IIDifr Pan F.

\section{Results}

Specimens of articular cartilage from 21 femorat heads were examined for fibronectin by in munoperoxidase staining. Four were specimens of normal (type I) femoral head cartilage from subjects aged between 12 and 65 years. Osteoarthrit specimens were taken from 17 subjects $(7$ men, women) aged between 34 and 79 (mean age years). Two of these patients had total hip replace ments for osteoarthritis which were secondary epiphyseal dysplasia and congenital dislocation the hip.

Fig. 1 illustrates schematically the classicat appearance of femoral heads removed at surgery for. osteoarthritis. Specimens which could not be oriens tated in this way, or where residual, type IV cartilage was not available at this lateral site, were not included. This ensured that all specimens? osteoarthritic and disease free, were comparab and that osteoarthritic cartilage was taken from joints at similar stages of degeneration.

The distribution of fibronectin in cartilage was examined mainly using immunoperoxidase staining Digestion of sections with chondroitinase $\mathrm{ABC}$ \% hyaluronidase (leech or testicular) was found 


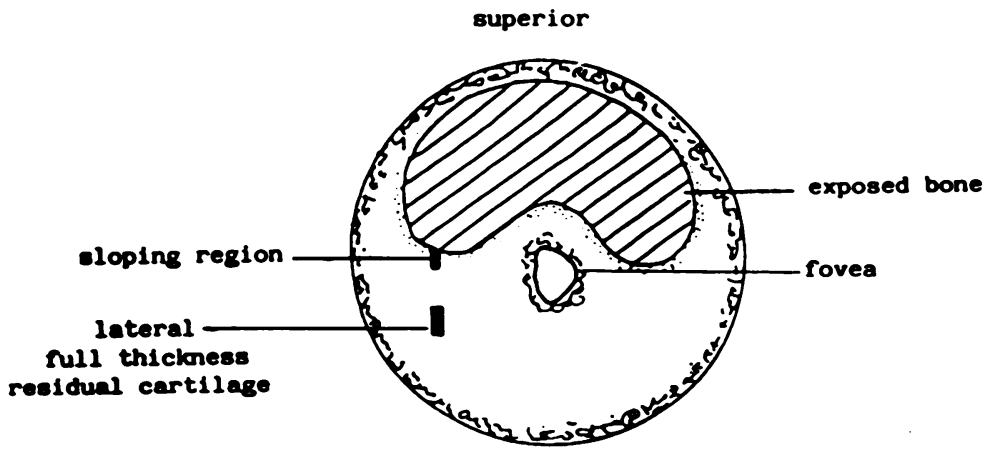

Fig. 1 Diagrammatic appearance of an osteoarthritic femoral head indicating the regions sampled for full depth and sloping residual cartilage.

intensify staining in all instances, though it was not essential to unmask antigen at the articular surface. Each enzyme treatment was effective in exposing the fibronectin antigen. Fluorescence staining indicated a similar distribution, chiefly in the surface zone (Fig. 2), though interpretation was complicated by autofluorescence of the cartilage.

In the cartilage matrix the most striking staining was seen at the surface of full depth osteoarthritic specimens (Fig. 3). A broken band of stain was seen in the upper $20 \%$ of all osteoarthritic specimens, the intensity and depth varying between specimens. Staining was not related to the surface fibrillation, as shown in Fig. 3b where a region of smooth cartilage was stained in the same way. Cells in the surface zone were frequently seen within islands of unstained matrix surrounded by antigen, either as a large zone or as a pericellular ring of fibronectin (Fig. 3d). Staining was very local with some cells lying in areas of strongly stained matrix adjacent to unstained cells and matrix.

Whereas all osteoarthritic cartilage specimens examined to date have contained surface fibronectin in varying quantities, none of the four disease free specimens contained significant amounts of fibronectin (Fig. 4). Two out of 17 osteoarthritic specimens also contained distinctive matrix staining in the mid to deep zone (Fig. 5). Stained areas were frequently elongated and patchy in appearance. No such patterns were seen in normal cartilage, which was invariably negative throughout the matrix. Osteoarthritic cartilage matrix from the slope region was normally stained through its full depth with local areas of heavy staining. Osteophytic cartilage (type VI) from osteoarthritic specimens contained no detectable fibronectin antigen in either the matrix or cells. Fibronectin was also undetectable in any region of normal, growing femoral head articular cartilage from a 12 year old individual.

Intracellular fibronectin antigen was seen less frequently than matrix fibronectin. Figs $6 a-c$ illustrate a group of surface cells which contained fibronectin, apparently located in tubular

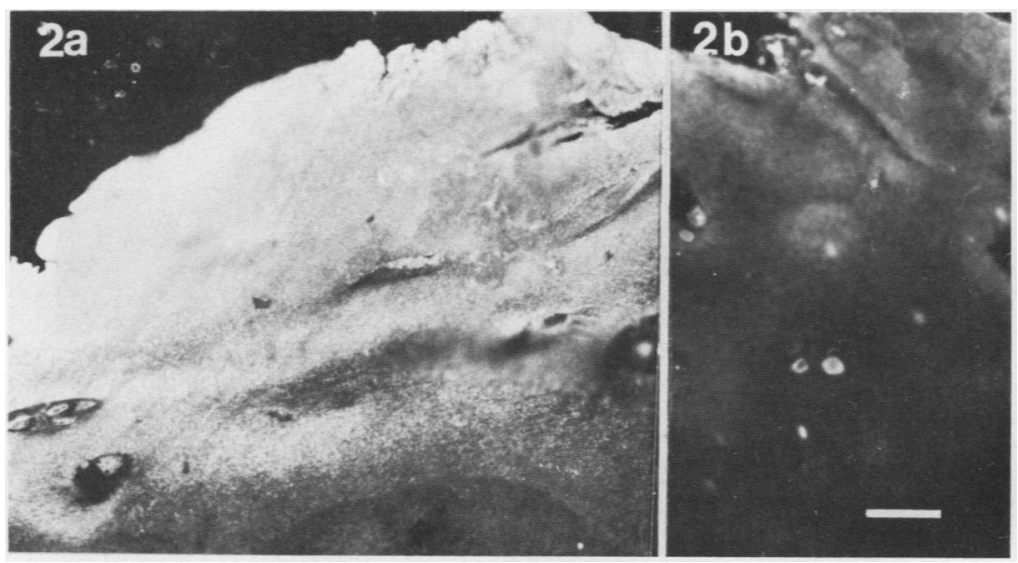

Fig. 2 Identification of fibronectin (2a) in full depth osteoarthritic cartilage (surface zone) by indirect immunofluorescence; $(2 b)$ control section, treated with non-immune sheep serum in place of sheep antihuman fibronectin. Note the localisation of antigen chiefly in the superficial region of the osteoarthritic cartilage matrix (2a). The control treatment of the same specimen (2b) showed only background staining. Bar $=50 \mu \mathrm{m}$. 
Fig. 3a

Fig. 3 (a) Fibrillated, full depth osteoarthritic cartilage with surface fibronectin stained by the immunoperoxidase technique. The patient was a 79 year old woman with primary idiopathic disease. Accumulations of fibronectin are present in the intercellular matrix in the surface zone. Staining was not continuous and in this case was most pronounced just below the surface. Pale staining persisted to a considerable depth.

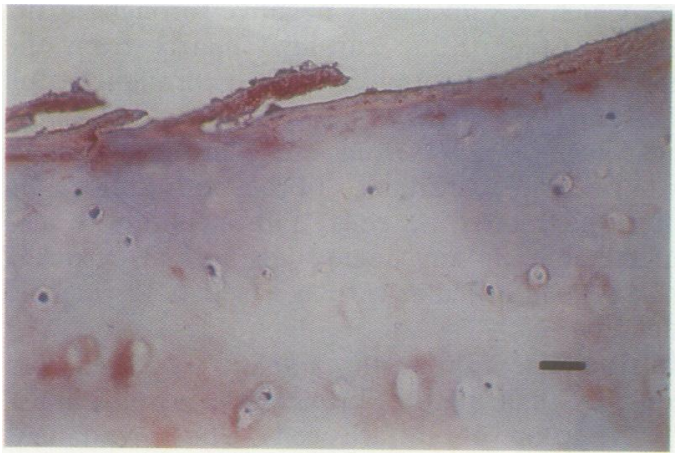

Fig. 3c

Fig. 3 (c) Osteoarthritic, surface zone cartilage stained lightly in the matrix but with marked pericellular accumulations as rings or polarised deposits (notably in the lower left hand corner).

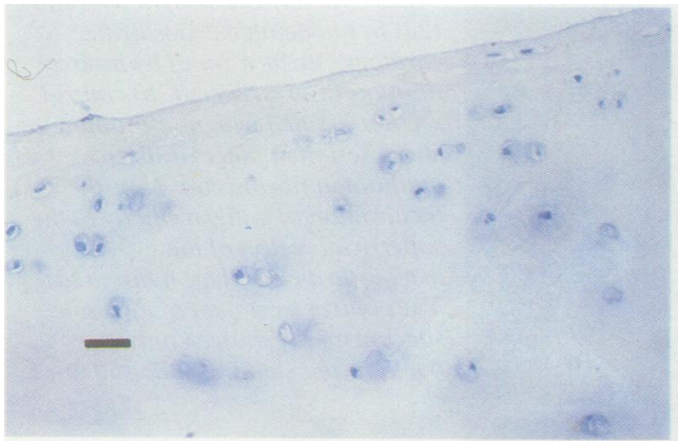

Fig. 4 Normal (type I) femoral head articular cartilage from a 46 year old male subject treated in the same way as in Figs $3 a-c$. Staining for fibronectin was negligible in both cells and matrix. Bar $=40 \mu \mathrm{m}$.

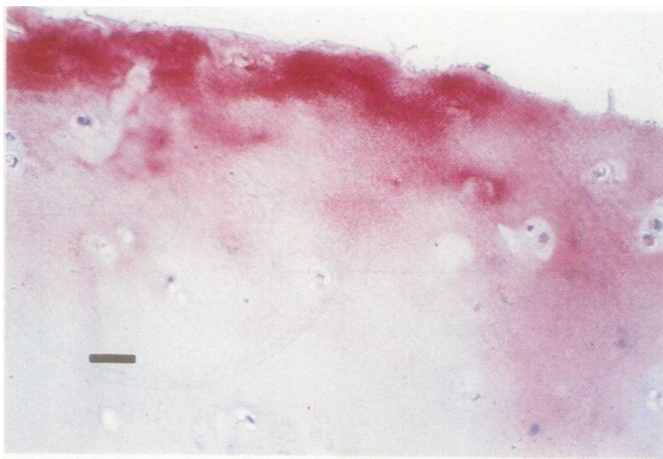

Fig. 3b

Fig. 3 (b) Non-fibrillated cartilage surface (same patient) containing a distribution of fibronectin similar to that seen in (a) despite the difference in surface integrity.

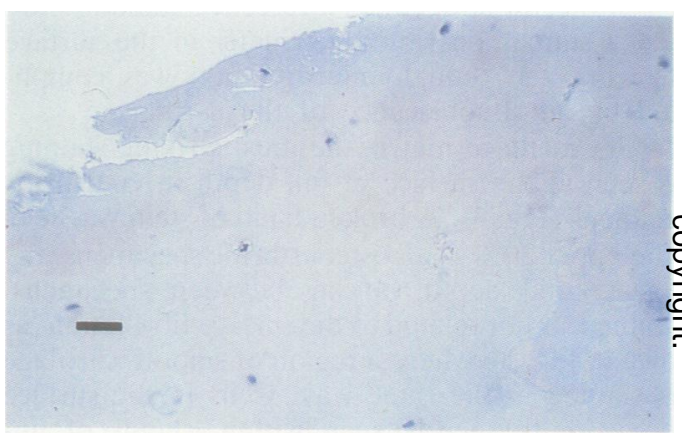

Fig. 3d

Fig. 3 (d) Control treatment of osteoarthritic cartilage specimen from the same patient. The section was treated with unconjugated antihuman fibronectin before incubation with antihuman fibronectin-peroxidase. Staining in the cell. and matrix was minimal.

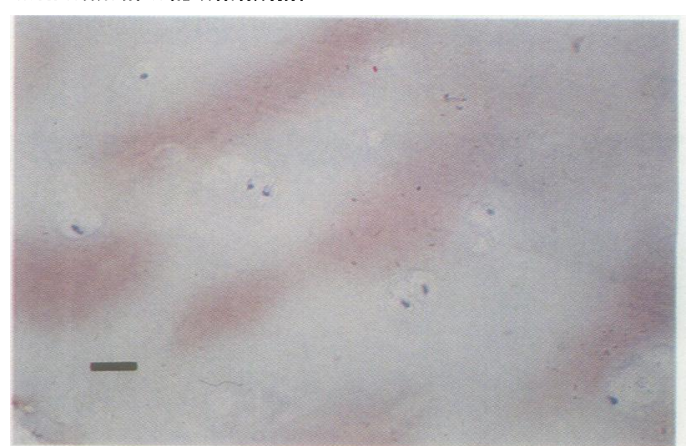

Fig. 5 Mid and deep zone of osteoarthritic cartilage showing localised deposition of fibronectin in the intercellular matrix. Staining in this region of full depth osteoarthritic cartilage was seen in only two specimens, both osteoarthritic. Intracellular or pericellular fibronectin was not present at this level in full depth cartilage. These deposit were quite separate and distinct from the surface accumulation of fibronectin and were not, apparently, part $\frac{?}{+}$ of the same distribution pattern. Orientation of the specimer 2 is the same as in Fig. 4 with the articular surface upwards. 우 Bar $=40 \mu \mathrm{m}$. 


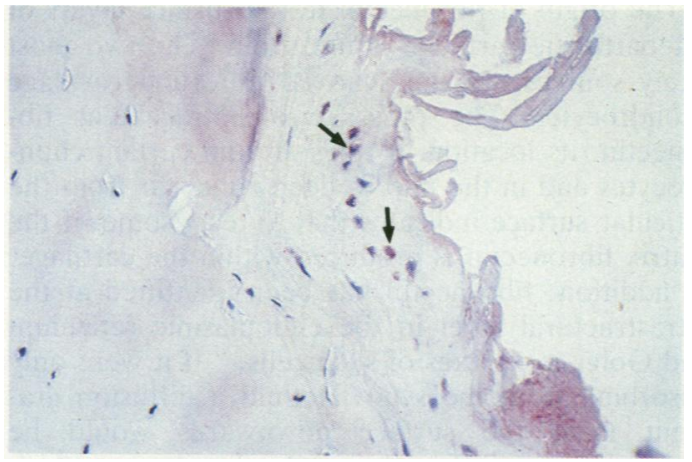

Fig. 6a

Fig. 6 (a) Intracellular immunoperoxidase staining in surface zone chondrocytes from fibrillated, full depth osteoarthritic cartilage (57 year old male patient with primary idiopathic osteoarthritis). Note that the group of stained cells (arrowed) are lying in unstained matrix.

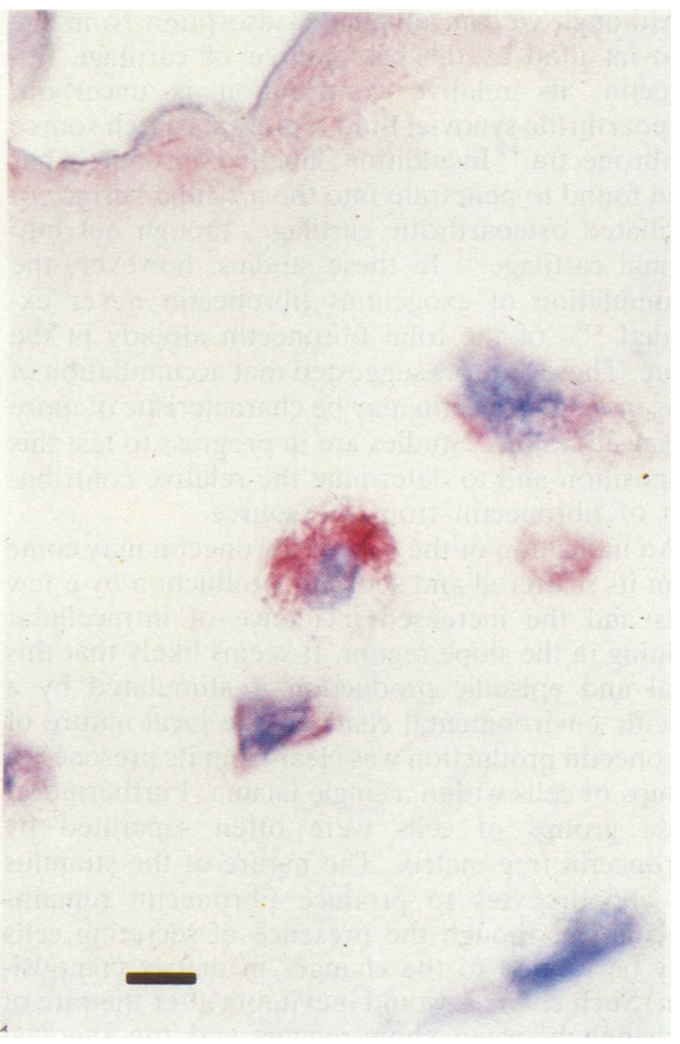

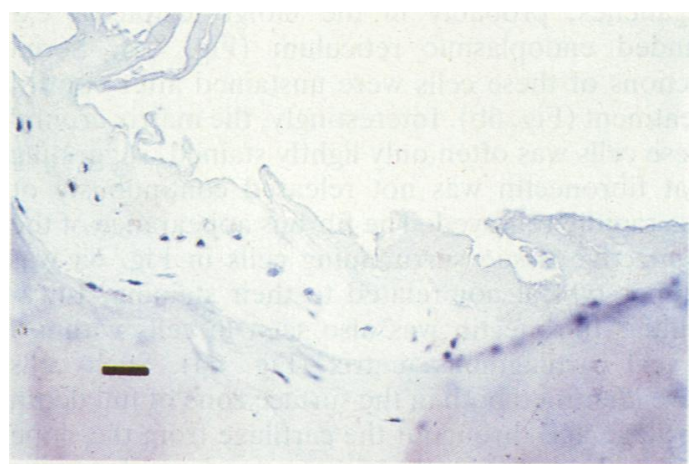

Fig. $6 \mathrm{~b}$

Fig. 6 (b) A control serial section from (6a) pretreated with unconjugated antihuman fibronectin to block fibronectin antigen on the specimen before incubation with antihuman fibronectin-peroxidase. Cells which stained for fibronectin in $6 a$ were unstained in this section. Bar $=40 \mu \mathrm{m}$.

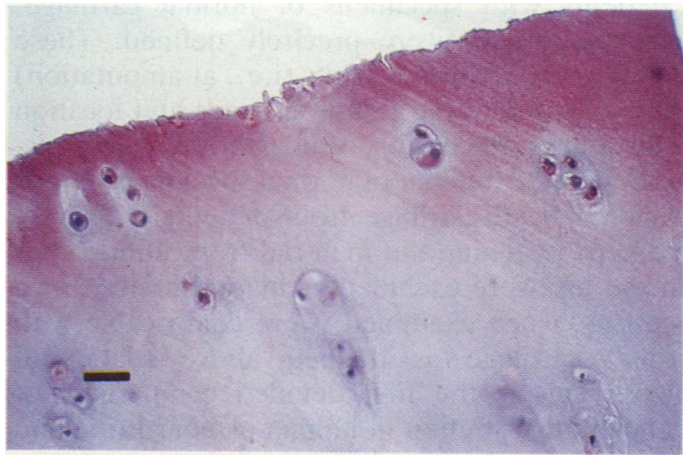

Fig. 6d

Fig. 6 (d) Intracellular staining of groups of cells at the surface of slope region cartilage (type IV). Bar $=40 \mu \mathrm{m}$.

Fig. 6 (c) High magnification of cells containing fibronectin, from $6 a$. Note the polarised distribution of stain within the cell and its apparent inclusion in tubular structures. Bar $=2 \mu \mathrm{m}$. 
organelles, probably in the Golgi region or expanded endoplasmic reticulum (Fig. 6c). Serial sections of these cells were unstained after control treatment (Fig. 6b). Interestingly, the matrix around these cells was often only lightly stained, suggesting that fibronectin was not released continuously or was rapidly removed. The fibrous appearance of the connective tissue surrounding cells in Fig. 6a was neither typical nor related to their staining. Intracellular fibronectin was also seen in cells within a typical cartilaginous matrix (Fig. 6d). Such cells were identified both in the surface zone of full depth cartilage and througout the cartilage from the slope region. In addition, there was a tendency for more than one cell within a lacuna to stain for fibronectin, suggesting that they may respond in localised groups.

\section{Discussion}

In this study we have compared the distribution of fibronectin in articular cartilage from 17 osteoarthritic patients with specimens of normal cartilage. Normal specimens were precisely defined. These were taken from normal joints (i.e., at amputation) matched for joint type (femoral head) and location on the joint surface with osteoarthritic specimens. This limited the number of normal specimens to four as femoral head cartilage from patients with fractured neck of femur and material from joints other than the hip were excluded as inappropriate. Such specimens, when examined, were comparable with 'normal' cartilage as defined above (data not shown). This is the first detailed comparison of fibronectin distribution in human osteoarthritic and normal cartilage at the cellular level. Immunoperoxidase localisation gave a more reliable identification of fibronectin in cells and matrix than immunofluorescent techniques, in agreement with Scott et al. ${ }^{15}$

Unlike previous workers, ${ }^{13}$ who found large quantities of fibronectin in non-osteoarthritic cartilage of rabbits and dogs, we were unable to detect fibronectin in non-osteoarthritic human cartilage. Shiozawa et al also failed to identify fibronectin in normal human articular cartilage. ${ }^{19}$ Our results correlate well with biochemical analyses from articular cartilage, which have shown that both human $^{7}$ and canine ${ }^{110}$ osteoarthritic cartilage contain far higher levels of fibronectin than non-osteoarthritic cartilage. This suggests that there is a connection between the presence of fibronectin antigen in the matrix and osteoarthritic cartilage changes. This is not consistent, however, with the suggestion that the presence of fibronectin in cartilage is specific for rheumatoid arthritis. ${ }^{19}$
The origin of fibronectin in the surface layers of osteoarthritic cartilage is uncertain. The two most: likely sources are the synovial fluid and cartilage chondrocytes. The presence of intracellular fibronectin, its location in rings around certain chon-등 drocytes and in the mid to deep zone, far from the articular surface indicates that at least some of thed matrix fibronectin is produced within the cartilage. In addition, fibronectin has been identified at the $e^{\infty}$ ultrastructural level in the endoplasmic reticulum $\vec{\odot}$ and Golgi complexes of such cells. ${ }^{20}$ If it were only $\vec{\overrightarrow{ }}$ adsorbing from the synovial fluid a diffusion gra- $\omega$ dient from the surface downwards would be expected ${ }^{21}$ rather than the patchy distribution which was observed. In addition, adsorption from the jointo cavity could not explain its presence in the cells, or $\overrightarrow{-}$ its localisation in rings around clones of chondro-o cytes seen in osteoarthritic specimens. Furthermore, radiolabelling studies have shown that cartilage explants can synthesise fibronectin in culture. ${ }^{11}$ -

Although we can eliminate adsorption from theo synovial fluid as the sole source of cartilage fibronectin, its relative contribution is uncertain $\frac{3}{0}$ Osteoarthritic synovial fluid represents a rich sourced of fibronectin. ${ }^{14}$ In addition, labelled fibronectin $\mathrm{kis}$ been found to penetrate into the articular surfacegpo fibrillated osteoarthritic cartilage, though not injonormal cartilage. ${ }^{21}$ In these studies, however, accumulation of exogenous fibronectin never ex-s ceeded $5 \%$ of the total fibronectin already in the tissue. These authors suggested that accumulation of exogenous fibronectin may be characteristic of more advanced lesions. Studies are in progress to test this $\overrightarrow{\vec{D}}$ proposition and to determine the relative contribu $\exists$ tion of fibronectin from the source.

An indication of the role of fibronectin may come. from its scattered and sporadic production by a feve cells and the increased incidence of intracellula? staining in the slope region. It seems likely that this local and episodic production is stimulated by specific environmental change. The local nature of fibronectin production was clear from its presence iक groups of cells within a single lacuna. Furthermore these groups of cells were often separated bo fibronectin free matrix. The nature of the stimulus for chondrocytes to produce fibronectin remains speculative, though the presence of secreting cells may be related to the changes in matrix composin tion. Such changes would inevitably alter the rate of exchange between chondrocytes and the synoviat fluid, particularly for large molecules. ${ }^{22}$ It has beeif reported that a loss of matrix proteoglycans car stimulate fibronectin production by chondrocytes. This may represent one mechanism for the initiation of its synthesis in osteoarthritic cartilage, where thळ levels of proteoglycan are reduced. ${ }^{23}$ Contrary tक्ष 
this, however, Wurster and Lust have reported that the depletion of proteoglycans alone is insufficient to stimulate fibronectin production in cartilage explants. ${ }^{24} \mathrm{We}$ are presently investigating the nature of such potential regulators. The absence of staining in immature, articular cartilage and osteophytic cartilage indicates that fibronectin is not produced by chondrocytes during cartilage growth and matrix production. No evidence has been found for a relationship between fibronectin production and cell shape.

Although these findings indicate that the accumulation of fibronectin is a feature of osteoarthritis, further studies will be necessary, using carefully defined clinical groups of patients, to determine whether its accumulation is pronounced in any particular group.

We are grateful to Mr John Collins for expert technical assistance, to Dr J R Ralphs for his helpful discussions, and to Miss Linda Adams for preparation of the manuscript. This work was supported by the Arthritis and Rheumatism Council.

\section{References}

1 Hynes R O. Fibronectin and its relation to cellular structure and behaviour. In: Hay E D, ed. Cell biology of extracellular matrix. New York: Plenum Press, 1981: 295-334.

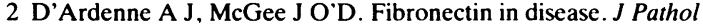
1984; 142: 235-51.

3 Yamagata M, Yamada K M. Yoneda M. Suzuki S. Kimata K. Chondroitin sulphate proteoglycan (PG-M-like proteoglycan) is involved in binding of hyaluronic acid to cellular fibronectin. $J$ Biol Chem 1986; 261: 13526-35.

4 Weiss R E, Reddi A H. Appearance of fibronectin during the differentiation of cartilage, bone and bone marrow. J Cell Biol 1981; 88: 630-6.

5 Evans H B, Ayad S, Abedin M Z, et al. Localisation of collagen types and fibronectin in cartilage by immunofluorescence. Ann Rheum Dis 1983; 42: 575-81.

6 Dessau W, Sasse J, Timpl R, Jilek F, von der Mark K. Synthesis and extracellular deposition of fibronectin in chondrocyte cultures. J Cell Biol 1978; 79: 342-55.

7 Miller D R, Mankin H J, Shoji H, D Ambrosia R D. Identification of fibronectin in preparations of osteoarthritic human cartilage. Connect Tissue Res 1984; 12: 267-75.

8 Clemmensen J, Holund B, Johansen N, Anderson R B. Demon- stration of fibronectin in human articular cartilage by an indirect immunoperoxidase technique. Histochemistry 1982; 76: 51-6.

9 Glant T T, Hadhazy Cs, Mikecz K, Sipos A. Appearance and persistence of fibronectin in cartilage. Histochemistry 1985; 82: $149-58$.

10 Wurster N B, Lust G. Fibronectin in osteoarthritic canine articular cartilage. Biochem Biophys Res Commun 1982; 109: 1094-101.

11 Wurster N B, Lust G. Synthesis of fibronectin in normal and osteoarthritic articular cartilage. Biochim Biophys Acta 1984; 800: $52-8$.

12 Wurster N B. Lust G. Deposition of fibronectin in articular cartilage of canine osteoarthritic joints. Am J Vet Res 1985: 46: $2542-5$.

13 Wurster N B, Butler M. Harter S, et al. Presence of fibronectin in articular cartilage in two animal models of osteoarthritis. $J$ Rheumatol 1986; 13: 175-82.

14 Scott D L, Farr M. Crockson A P. Walton K W. Synovial fluid and plasma fibronectin levels in rheumatoid arthritis. Clin Sci 1982; 62: 71-6.

15 Scott D L. Wainwright A C. Walton $\mathrm{K}$ W. Williamson $\mathrm{N}$. Significance of fibronectin in rheumatoid arthritis and osteoarthritis. Ann Rheum Dis 1981; 40: 142-53.

16 Lavietes B B, Carsons S. Diamond H S, Laskin R S. Synthesis. secretion and deposition of fibronectin in cultured human synovium. Arthritis Rheum 1985; 28: 1016-26.

17 Ali S Y, Bayliss M T. Enzyme changes in human osteoarthritic cartilage. In: Ali S Y, Elves M W, Leaback D H, eds. Normal and osteoarthritic articular cartilage. London: Institute of Orthopaedics, 1974: 189-217.

18 Johnson G D. Davidson R S. McNamee K C. Russell G, Goodwin D, Holborow E J. Fading of immunofluorescence during microscopy: a study of the phenomenon and its remedy. J Immunol Methods 1982; 55: 231-42.

19 Shiozawa K. Shiozawa S, Shimizu S, Fujita T. Fibronectin on the surface of articular cartilage in rheumatoid arthritis. Arthritis Rheum 1984; 27: 615-22.

20 Rees J A, Ali S Y, Brown R A. Ultrastructural localisation of fibronectin in human osteoarthritic articular cartilage. Ann Rheum Dis 1987; 46: 816-22.

21 Wurster N B, Lust G. Incorporation of purified plasma fibronectin into explants of articular cartilage from disease free and osteoarthritic canine joints. J Orthop Res 1986; 4: 409-19.

22 Maroudas A. Distribution and diffusion of solutes in articular cartilage. Biophys J 1970; 10: 365-79.

23 Bayliss M T, Venn M. Chemistry of human articular cartilage. In: Maroudas A. Holborow E J, eds. Studies in joint disease. Vol 1. Tunbridge Wells: Pitman Medical, 1980: 2-58.

24 Wurster N B, Lust G. Fibronectin in osteoarthritic cartilage-A possible indication of phenotypic modulation of the chondrocyte? In: Verbruggen G, Veys E M, eds. Degenerative joints. Vol 2. Amsterdam: Elsevier, 1985: 141-7. 